19 Revue d'histoire du XIXe siècle

Société d'histoire de la révolution de 1848 et des

révolutions du XIXe siècle

25 | 2002

Le temps et les historiens

\title{
Les temps retrouvés de Waterloo
}

Jean-Marc Largeaud

URL : http://journals.openedition.org/rh19/431

DOI : 10.4000/rh19.431

ISSN : $1777-5329$

Éditeur

La Société de 1848

Édition imprimée

Date de publication : 1 décembre 2002

Pagination : 145-152

ISSN : 1265-1354

Référence électronique

Jean-Marc Largeaud, "Les temps retrouvés de Waterloo », Revue d'histoire du XIXe siècle [En ligne],

25 | 2002, mis en ligne le 29 juin 2005, consulté le 20 avril 2019. URL : http://journals.openedition.org/ rh19/431 ; DOl : 10.4000/rh19.431

Ce document a été généré automatiquement le 20 avril 2019

Tous droits réservés 


\title{
Les temps retrouvés de Waterloo
}

\author{
Jean-Marc Largeaud
}

1 À qui entreprend de parler de Waterloo, le répertoire de la culture française fournit des références devenues inévitables. La facilité pourrait consister ici à rappeler, par exemple, Stendhal et l'interrogation de Fabrice del Dongo: "était-ce une véritable bataille" ? Le "syndrome de Fabrice" qui a été déduit du célèbre chapitre de La Chartreuse de Parme est resté une figure de l'incompréhension de n'importe quel phénomène social ou historique. Pour l'avoir retrouvé sous la plume de plusieurs historiens français du vingtième siècle, de Raymond Aron à Maurice Agulhon ou Michel Vovelle ${ }^{1}$, faudrait-il n'en retenir que l'usage d'un lieu commun, la longue durée des faits de langage ou le poids de la littérature dans la formation des historiens? Ou, plus imprudemment, devra-t-on aussi trouver dans la force singulière du "syndrome" la trace des vertus critiques et/ou de la séduction de l'ironie de Stendhal?

2 Substituons une facilité à une autre, car les historiens ne lisent pas uniquement des romans. En 1834, cinq ans avant la parution de La Chartreuse de Parme, un manuel d'histoire demandait aux lecteurs de répondre à une question dont la brièveté, la qualité pédagogique et la tonalité inquiète peuvent aujourd'hui laisser pensif: "Dites quelque chose de la bataille de Waterloo" ${ }^{2}$. De l'injonction qui précède déduira-t-on la vérité de ce que les Français d'alors cherchaient à dire du désastre : "quelque chose" ?

3 Entre l'inscription dans l'histoire élémentaire et la vision romanesque, on admettra provisoirement ici que l'injonction possède la vertu heuristique attendue d'un programme de recherche contemporain. On peut l'envisager dans un sens classique, car chercher ce qu'a représenté la défaite de Waterloo pour les Français du XIX ${ }^{\mathrm{e}}$ siècle ${ }^{3}$ revient à s'interroger sur la façon dont l'événement est perçu ou utilisé. Plus largement, il convient de se demander si un événement est pensable pour un historien en dehors des représentations qui le constituent comme événement, ce qui n'est pas seulement construire un objet et rendre compte de sa chronologie.

4 L'étude abordera d'abord les questions posées par le temps court de l'événement. Dans la seconde partie, il sera fait état du destin surprenant de Waterloo à l'intérieur du temps politique au cours du XIX siècle. Dans la mesure où la longue durée de la défaite du 
18 juin 1815 invite à analyser l'interrogation rétrospective des Français avant 1914, on évoquera dans la dernière partie l'utilité et les difficultés d'une relecture au second degré de l'événement.

5 L'étude attentive des sources autorise à se demander ce que fut, en son temps, l'événement. La nouvelle de la défaite de Waterloo a précédé l'annonce officielle du désastre par Napoléon. Les conditions dans lesquelles les bruits et les rumeurs se propagent n'ont pas de précédent, même si on peut évoquer la situation dramatique de 1793. Le bruit de la défaite se manifeste dès le 19 juin ${ }^{4}$, sur les axes de retraite de l'Armée du Nord et même beaucoup plus à l'écart ${ }^{5}$. De plus, la "vérité" impériale, diffusée dans Le Moniteur du 21 juin 1815, constitue une nouveauté sans équivalent dans l'histoire de France, en dépit de l'existence du XXIX ${ }^{\mathrm{e}}$ Bulletin qui présentait au pays les désastres de Russie en 1812. La nouvelle, annoncée par l'Empereur lui-même, dans un pays divisé et menacé, en proie à l'inquiétude, révèle, dans sa brutalité, la réalité de l'événement. La force en est redoublée dans les régions qui commencent à subir une invasion souvent brutale. La défaite est donc connue, suivant les lieux, entre le 19 et le 27 juin (au plus tôt) ; elle précède de peu l'annonce de l'abdication.

6 La sobriété du constat pourrait ici suffire, mais laisse insatisfait. Il est pourtant difficile d'aller plus loin car beaucoup d'incertitudes demeurent sur la diffusion de la nouvelle et sur les réactions immédiates. Il faut ainsi regretter le quasi silence des responsables politiques et militaires dans les archives conservées: on peut prudemment émettre l'hypothèse d'une destruction des documents avant le retour de Louis XVIII ${ }^{6}$... Il est par conséquent difficile ou hasardeux de faire à la suite de Henri Houssaye le tour de France des réactions à la défaite ${ }^{7}$. Ce que l'on en connaît est fragmentaire ${ }^{8}$ et, curieusement, l'historiographie française a longtemps délaissé ${ }^{9}$ cette période trouble de l'été 1815 , sauf dans les revues d'histoire locale ${ }^{10}$. Faudrait-il conclure à l'indifférence ou à la neutralité face à l'événement?

7 L'étude de la presse pourrait le laisser croire en partie. Elle représente une des vraies curiosités de ces temps de guerre et de défaite. Que publie-t-on en effet sur Waterloo? Entre le 21 et le 27 juin Le Moniteur fournit divers récits de la bataille. Se succèdent Napoléon, le général Drouot, le maréchal Ney dans son intervention à la Chambre des pairs le 22 juin, et plus curieusement, la traduction de la relation de Wellington. En ajoutant la lettre de Ney à Fouché publiée dans le Journal de l'Empire du 27 juin, la liste des principaux récits contemporains est complète. Elle sert de base documentaire à une presse qui commente fort peu l'événement et se contente de le reproduire ou d'y faire allusion. Les maigres articles des journalistes méritent de retenir pourtant l'attention. D'abord parce que fort peu de place est donnée à l'ennemi comme menace ou même comme fantasme. Ensuite parce que sont fournis des détails fantaisistes ou des commentaires promis à l'oubli (l'indication que les soldats de la Garde faits prisonniers auraient été systématiquement fusillés), ou destinés à la plus grande postérité (la phrase fameuse attribuée à Cambronne apparaissant dès le 29 juin). Enfin, parce qu'on peut y lire la réapparition d'une partie de la rhétorique nationale issue de la Révolution, forme de réaction à la défaite, sans commune mesure avec la détermination de 1793. En admettant le poids des incertitudes politiques et militaires, la rapidité de la Restauration laisse en fait peu de chances de saisir l'éventail et surtout la sincérité des réactions du moment.

Mais il serait périlleux de borner le temps court de l'événement à l'effondrement brutal de juin et juillet 1815 et à l'expectative des contemporains, car l'événement prend corps dans plusieurs séries de traditions picturales ou de filiations de textes où s'élaborent, 
dans le cadre d'une reconstruction, les premières représentations de la défaite. Il est présent dans les images qui ne dureront pas, celles de la légende noire, comme dans les images qui resteront : les grenadiers, Cambronne, le dernier carré ${ }^{11}$. Il est interprété dans des textes dont on retiendra celui de Pierre Colau ${ }^{12}$, polygraphe obscur dont le récit, publié dès août 1815 , fondé sur une combinaison des relations de Napoléon et du général Drouot tirées du Moniteur, servira de base à plus d'un tiers des relations de la bataille jusqu'à la fin de la Monarchie de Juillet. Si le livre de Colau relève de la catégorie des classiques de l'ombre souvent plagiés, il n'en est pas de même pour celui de Casimir Delavigne dont Les Messéniennes paraissent en $1818{ }^{13}$. Cette entreprise de consolation de la France vaincue au moment où les Alliés quittent le territoire livre sans doute un jalon de la chronologie de l'histoire de Waterloo, d'une bataille perdue aux réalités matérielles de l'occupation d'une partie du pays.

Waterloo demeure dans le temps long: il est nécessaire de retrouver les relations spécifiques de l'événement avec l'histoire.

De Delavigne en 1818 à Henri Houssaye en 1898, dernier grand succès de librairie pour "Waterloo" au XIX siècle, la logique voudrait que l'on ne considère que les registres de l'histoire culturelle ou de l'historiographie pour rendre compte de la longue durée de l'événement. Avec un peu de bonne volonté, il faudrait en outre préciser qu'entre les deux dates s'exprime de façon différente la solide et durable tradition de l'anglophobie française, de Waterloo jusqu'à Fachoda. Mais ce serait méconnaître d'une part la coupure fondamentale de 1870 et d'autre part laisser dans l'ombre un des enseignements de ce que furent d'abord, mais pas uniquement, les temps de Waterloo: ceux de la politique intérieure et de ses surprenantes appropriations de l'événement, non ceux d'une véritable volonté de revanche ${ }^{14}$.

11 Avant 1870, Waterloo est un élément parmi d'autres de la rhétorique nationale et du discours sur la Nation. Il est en général utilisé par des opposants afin de dire qui a le droit de parler au nom de la "Nation" ou de la "Patrie" et de ses intérêts pour disqualifier l'adversaire au pouvoir. Revendiquer la grandeur et l'héroïsme des vaincus va de pair avec la volonté de se montrer plus national que l'adversaire politique. Libéraux de l'opposition à Louis XVIII, membres du "mouvement" et légitimistes sous la Monarchie de Juillet, républicains sous tous les régimes ont donc brandi l'épouvantail de Waterloo. Si l'on admet la logique des appropriations politiques, c'est bien une volonté de contrôle politique de la référence à la bataille qui s'énonce dans la revendication. Or, les différents pouvoirs mis en cause ne sont pas restés l'arme au pied. Leurs réponses ont oscillé entre une répression très modérée avant 1830, et la réponse symbolique après cette date. Parce qu'il est impossible de revendiquer ou de commémorer la défaite, la réplique est donc de l'ordre de la stratégie indirecte. Sous Louis-Philippe, le programme de décoration de l'Arc de triomphe réalisé en 1836 constitue le meilleur exemple de choix symbolique. Waterloo s'inscrit en filigrane entre la mention de la dernière victoire, Ligny, et l'allégorie de "la Paix" (1815), faisant pendant à l"'Invasion" pour 1814. Sous le Second Empire, en fonction de ses propres objectifs et sous le feu de la critique républicaine, la propagande du pouvoir jouera sur les succès extérieurs et sur la reconnaissance de la Nation envers les soldats pris dans la tourmente des guerres de 1792 à 1815 . Elle honorera tout spécialement les soldats du bataillon de l'île d'Elbe et les blessés de Ligny et de Waterloo lors du règlement du testament de Napoléon (1854-1857). On en conclura que, par une présence en arrière-plan, Waterloo peut aussi servir l'ordre établi. 
12 Après 1870, après Sedan, Waterloo aurait dû disparaître. Il n'en est rien même si la référence est maintenant située ailleurs que dans le champ des luttes politiques. La récupération de la bataille par les républicains qui en font délibérément un élément essentiel de la culture scolaire républicaine suggère cependant davantage qu'un déplacement du terrain. Avec la condamnation du césarisme menant à la défaite, la place de Waterloo dans l'éducation des citoyens est fondamentale chez Lavisse et d'autres pédagogues. Elle dévoile que les républicains avaient --et pour cause-- intériorisé et neutralisé la fonction de contestation rattachée à Waterloo.

Avant 1914, l'événement marquant est en 1898 l'histoire d'Houssaye. Elle est appréciée pour ses qualités dramatiques mais aussi parce qu'elle se rapproche des objectifs scientifiques de la discipline à la fin du XIX ${ }^{e}$ siècle : utilisation massive des sources (et des notes infrapaginales), érudition et souci de la preuve. Il faudrait alors utiliser la catégorie d'événement culturel pour rendre compte d'un triomphe de la librairie. Mais ce n'est qu'une des composantes d'un succès qui lui vaut les louanges de la gauche (et de Jaurès) comme de l'extrême droite (qui voit dans l'œuvre la "démonstration saisissante" du "nationalisme intégral" de Maurras ${ }^{15}$ ). On peut interpréter cette unanimité comme l'expression d'une consolation après Fachoda, donc comme l'adhésion à une expression historiographique de la notion de "revanche". Mais on doit se demander si la présence de Waterloo au moment où se déroule l'affaire Dreyfus ne donne pas la clé du véritable contexte et une explication possible: Waterloo fut à la fois une façon de dire et d'exorciser "l'esprit de guerre civile" ${ }^{16}$.

Décrite en fonction de mécanismes d'appropriation ou de contestation, la notion de "temps politique" peut prêter à confusion. Waterloo s'inscrit parfois dans les coupures traditionnelles de l'histoire de France, mais plus souvent dans un décalage à l'intérieur du temps politique, en-deçà ou au-delà des dates de naissance des nouveaux régimes ou des événements qui ramènent l'attention sur le 18 juin 1815. Il est ainsi des dates immanquables, celle de 1829 avec la célèbre attaque de Barthélémy et Méry contre Bourmont sous le ministère Polignac, celle de la révolution de 1830 considérée comme "le dernier coup de canon de Waterloo" dans une formule revendiquée par Alexandre Dumas. Il en est d'autres dont la signification et l'importance doivent être reconstruites. L'année 1857 peut être retenue dans la chronologie pour la parution des Châtiments de Hugo et la publication à Bruxelles de l'Histoire de la campagne de 1815 sous la plume du lieutenantcolonel Charras, véritable chef de l'opposition républicaine en exil: Waterloo s'insère dans la pensée critique républicaine. Or, la place dans l'histoire de l'événement ne prend tout son sens qu'en fonction d'un autre élément du contexte. En 1857, en effet, Napoléon III réinvente le stéréotype du vieillard glorieux ayant bien mérité de la Nation avec la médaille de Sainte-Hélène qui récompense les anciens combattants de la République et de l'Empire. Et si l'on souhaite prouver que l'intervention des républicains en 1857 représente un tournant de cette histoire, il faut tenter l'expérience en dehors des seules indications de contenu et retrouver la généalogie de l'implication de Hugo ou de Charras. C'est alors qu'une périodisation s'impose. On justifiera dans ce cas les dates limites de 1848 et 1857 en évoquant les séquelles de Juin 1848 qui font de l'inauguration du monument de Cambronne à Nantes une opération de maintien de l'ordre, l'achat d'un tableau sur Waterloo par le Comte de Chambord en 1850 ou encore les fortes paroles de Thiers désignant le parti de l'ordre comme "vaincu de Waterloo" ${ }^{17}$. L'histoire de Waterloo est ainsi située dans l'évolution politique qui mène à l'échec final de la République. Il faut 
en tirer la conclusion : les républicains exilés pensent Waterloo en fonction du coup d'état de 1851 et des divisions issues de juin 1848 .

Or, l'exemple du livre de Houssaye le suggère, seul le croisement constant de sources d'origines diverses permet de reconstituer l'ensemble de la chronologie sur le temps long du XIX ${ }^{e}$ siècle, en précisant les données directement issues du temps de la politique et en justifiant les inflexions d'une histoire de l'événement. Waterloo montre une façon de faire de la politique "avec d'autres moyens". Mais l'obligation de rendre compte d'une part de la diffusion ou de l'impact du souvenir et des thèmes rattachés à la bataille, d'autre part des images ou de la grande peinture et des textes isolés, ouvre sur de considérables difficultés d'analyse.

Ressaisir l'événement dans sa globalité exige de retrouver les milieux, les processus et les règles où il se constitue et s'énonce. Il convient donc d'admettre comme indispensables l'attention aux vecteurs de la mémoire, à la résurrection des codes culturels et aux relations entre identités et événement.

Qui porte la mémoire de Waterloo ? Avec le cumul d'un effet de sources et des difficultés de la recherche, on atteint au mieux des groupes, des tendances politiques, des réactions d'individus instruits. Il n'est possible d'entrevoir des perceptions populaires de l'événement que de façon indirecte, dans le Paris de 1830 par exemple. Les autobiographies "populaires" renseignent mal sur ce qu'a pu représenter Waterloo et il serait hasardeux d'extrapoler à partir des remarques incidentes de Norbert Truquin sur la bataille. Des fragments d'interprétation délicate existent. Par exemple, les dossiers des médaillés de Sainte-Hélène mettent toujours en relief la participation aux combats de l'Armée du Nord et de 1815, et mentionnent parfois "Waterloo" même si le militaire était parfois loin du théâtre des combats de 1815. Mais la médiation --et les commentaires-- des maires ou d'autres autorités laissent penser qu'il s'agit au moins autant d'une mémoire confisquée que d'une mémoire populaire... Malgré les incertitudes sur l'oralité et la transmission des thèmes napoléoniens, il conviendrait donc de s'interroger sur les problèmes d'histoire sociale qui justifient la longue durée de l'événement et sur leur propre temporalité.

Pour étoffer une analyse des vecteurs de mémoire, est-il davantage pertinent de se tourner vers les ressources spécifiques de l'histoire culturelle ? À s'en tenir à l'histoire du livre les résultats ne sont pas négligeables. Dans la littérature historique exclusivement ou largement consacrée à la campagne de 1815 , le républicain Achille de Vaulabelle devance tous ses concurrents avec 16000 exemplaires et huit éditions de l'Histoire des deux Restaurations de 1844 à 1874 et deux récits de la campagne de 1815 publiés à part ${ }^{18}$. Il est rejoint par Thiers avec le vingtième volume de l'Histoire du Consulat et de l'Empire et Waterloo tiré à part en 1862 à 10000 exemplaires. Le grand succès historique du siècle revient incontestablement à Henri Houssaye, dont l'ouvrage fut édité à 75000 exemplaires de 1898 à la guerre de 1914. Il est légitime de les comparer à d'autres: Le Mémorial de Sainte-Hélène de Las Cases (40 000 exemplaires au moins avant 1850), Les Misérables et Les Châtiments (91000 et 60000 exemplaires) de Victor Hugo, le Waterloo d'ErckmannChatrian (59915 exemplaires). Mais en dehors du palmarès, de l'esquisse d'une topographie éditoriale particulière et de la confirmation de la chronologie et du poids de la politique, l'histoire de l'édition ne permet pas de ramener de façon univoque au contenu de ce qui a été réellement connu --ou dit-- de l'événement.

À défaut, la recherche des codes culturels ouvre d'autres perspectives. Toute bataille, et tout événement, "dignes de mémoire", possèdent des codes culturels dans lesquels ils 
s'inscrivent et/ou qui leur sont propres. Il s'agit de trouver les registres de l'explication et de comprendre la place de Waterloo dans les représentations, donc dans des temporalités différentes. Il importe de déterminer ce qui est historiquement actif, ce qui éclaire spécifiquement l'histoire au second degré de la bataille dans les idéologies ou les mentalités collectives. L'étude du rapport à la guerre, de l'héroïsme, de l'histoire militaire ${ }^{19}$ et même celle des "mots" de Waterloo trouve alors sa justification.

Ce type d'approche fait encourir à juste titre le reproche d'un empirisme mal maîtrisé. Or la fréquentation studieuse des textes conduit à élaborer un éclairage récurrent qui interdit de prendre systématiquement des répétitions pour des reproductions à l'identique de sentiments, d'images ou d'analyse. Les mécanismes identitaires à l'œuvre dans l'expression de la relation à Waterloo conduisent à exploiter ce qui est un peu plus qu'une hypothèse de travail. Les identités explicitement mises en rapport avec Waterloo sont exprimées de deux manières complémentaires. En premier lieu, écrire sur la bataille est une forme d'expression de soi. Contemporains de l'événement, mémorialistes, poètes et historiens se conforment à l'idée d'une obligation : dire "quelque chose" de personnel (le plus souvent d'une banalité sans bornes) de la chute de Napoléon et de la Grande Armée. Nul ne l'a mieux réussi que Chateaubriand dans un passage célèbre où l'auteur guette les bruits de la bataille et réfléchit sur des sentiments probablement reconstruits. Dans les Mémoires d'Outre-Tombe, l'auteur écrit en effet: "la patrie l'emportait en ce moment dans mon cœur" " ${ }^{20}$. Ce procédé d'association du nom de la bataille ou des mots "France" ou "patrie" ou "liberté" au "moi" est général et se retrouve chez tous ceux qui ont cédé à la tentation de faire de "leur" Waterloo, un hommage à la mesure de la fierté déçue, un morceau de bravoure inégalement réussi.

De plus, Waterloo est un réceptacle pour d'autres identités que celle de "Français". L'intériorisation se pratique dans la posture et l'argumentation de diverses tendances de vaincus. On peut légitimement en rappeler l'énumération déjà entrevue : battus de "1830" (légitimistes, puis républicains), républicains victimes du 2 décembre 1851, anciens combattants de $1870 \ldots$ Il faut par conséquent admettre que plusieurs générations de vaincus peuvent s'identifier aux héros malheureux de Waterloo. Cette intériorisation est d'ailleurs confirmée par le rappel ou la revendication de Waterloo chez des minoritaires ou des marginaux. Waterloo sert à exprimer l'inquiétude devant le bouleversement social, à dénoncer la pathologie urbaine ou l'insolente fortune des banquiers Rothschild. On s'identifie donc positivement aux vaincus du 18 juin pour des raisons qui peuvent rappeler les problèmes spécifiques de 1815 ou, à la lettre, n'en rien dire.

La force d'attraction de la grandeur dans la pire des adversités, de la défaite glorieuse, a ainsi donné naissance à une "culture de la défaite" sous la pression d'autres événements qui lui donnent sa propre chronologie. Pour l'ensemble du XIX ${ }^{e}$ siècle et peut-être pour le $\mathrm{XX}^{\mathrm{e}}$, Waterloo lui fournit un leitmotiv et en fonde les ambivalences redoutables, entre délectation morose, velléités de revanche et résignation.

Les Français du XIX ${ }^{e}$ siècle ont à diverses reprises donné des lectures contradictoires ou concordantes de la bataille du 18 juin 1815 car les rapports de l'événement avec le discours sur la Nation, avec l'universel politique et de multiples identités ont garanti sa pérennité. La difficulté principale est de retrouver les repères et les césures d'un processus complexe, négatif et positif, de construction d'une temporalité spécifique où la défaite est insérée dans la mémoire nationale par le biais des oppositions politiques. La manière dont l'événement est adopté ou revendiqué par les familles politiques ou les individus en a fait un élément d'identité partagée qui ne peut être analysé qu'en fonction 
d'un ensemble de représentations dont le contenu et la structure évoluent à l'intérieur de symboliques et de chronologies politiques, identitaires, guerrières ou nationales. Du temps court de l'événement au temps long des appropriations et des représentations se sont donc indissolublement tissé les liens de la formation conjointe d'une culture et d'un de ses thèmes, Waterloo, à la fois expérience et image définitive de la défaite glorieuse.

Jean-Marc Largeaud est maître de conférences

à l'Université François Rabelais de Tour

\section{NOTES}

1.. On nous pardonnera ici de ne pas faire de renvoi à des références qui seraient en dehors du contexte de la réflexion des historiens cités.

2.. L. C., Cours d'Histoire, Paris, Éditions Moronval, 1834, p. 275.

3.. Comme il ne sera pas fait de renvoi systématique aux allusions et aux citations dans la suite du texte, renvoyons à notre Waterloo dans la mémoire des Français (1815-1914), Thèse d'histoire sous la direction de Claude-Isabelle Brelot, Université Louis Lumière-Lyon 2, janvier 2000, 3 volumes.

4.. Par exemple à Laon.

5.. Le jeune Edgar Quinet apprend la nouvelle par des récits oraux, mais écrit en 1858 : “ Waterloo nous apparut en réalité par les premiers soldats débandés" passant à Charolles. Encore faudrait-il connaître les dates approximatives de l'arrivée des uns et des autres. Voir Edgar QUINET, Histoire de mes idées, Paris, Éditions Flammarion, 1972 (1 ${ }^{\text {ère }}$ édition 1858), pp. 106-107, souligné par nous.

6.. Les résumés des dépêches établis pour le ministère de l'Intérieur ont presque tous disparu pour les deux dernières semaines de juin 1815.

7.. Henri HOUSSAYE, 1815, tome 2, Waterloo, Paris, Librairie Plon, 1898.

8.. Rappelons les émeutes de Bourges contre les royalistes et le clergé (23-24 juin 1815) ou le déclenchement des prodromes de la Terreur Blanche à Marseille (24-27 juin).

9.. Pour la date de parution et la qualité du texte, il est cependant juste et opportun de rappeler le livre de Félix PONTEIL, La chute de Napoléon I ${ }^{\text {er }}$ et la crise française de 1814-1815, Paris, Éditions Aubier-Montaigne,1943, 349 p.

10.. Signalons au passage le grand silence des histoires régionales des années récentes, sauf exception.

11.. Le renouvellement des images n'apparait que plus tard, après 1830, où Napoléon revient dans le carré de la Garde, et avec Les Misérables qui relancent la cavalerie à l'assaut du Mont Saint Jean...

12.. Pierre COLAU, Histoire de Napoléon, Paris, Éditions Vauquelin, 1815.

13.. Une des Messéniennes est consacrée à Jeanne d'Arc ; voir Casimir DELAVIGNE, Trois Messéniennes, élégies sur les malheurs de la France, Paris, Éditions Ladiscat, 1818, 31 p.

14.. Qu'il y ait eu des âmes ardentes prêtes à en découdre, nul n'en doutera. Mais, qui, après 1815 , aurait pu raisonnablement prendre la responsabilité d'assumer la logique ultime de la revanche : l'acceptation d'un risque de guerre générale pour reprendre la 
Belgique et les frontières du Rhin ? Les républicains et les bonapartistes une fois au pouvoir après 1848 s'en sont bien gardés.

15.. Voir Le Soleil, 31 janvier 1901.

16. Voir Maurice AGULHON, "Pour une conclusion", dans Jean-Clément MARTIN [dir.], La Guerre civile, entre histoire et mémoire, Nantes, Ouest Éditions, 1995, p. 247.

17. Dans le célèbre discours sur la loi électorale de 1850, Le Moniteur, 24 mai 1850, p. 1805.

18. Achille de VAULABELLE, La Campagne et bataille de Waterloo, Paris, Perrotin, 1845, 219 p., est tiré à 5000 exemplaires en 1845 ; voir Archives nationales, F18* II 32.

19.. Nous renvoyons à Jean-Marc LARGEAUD, “Mémoire et identités : Waterloo et la genèse de la "défaite glorieuse" ", dans Natalie PETITEAU [dir.], Territoires, pouvoirs et identités : voies de renouvellement de l'histoire de l'Empire, colloque, Université d'Avignon, mai 2000, à paraître.

20.. Voir François-René de CHATEAUBRIAND, Mémoires d'Outre-Tombe, Bibliothèque de la Pléiade, Paris, Éditions Gallimard, 1946, livre 23, chapitre 16, p. 963. 